\title{
CMS Forward Calorimetry R\&D for Phase II Upgrade
}

\author{
Burak Bilki ${ }^{* \dagger}$ \\ University of Iowa, Iowa City, IA 52242 USA \\ Argonne National Laboratory, Argonne, IL 60439 USA \\ E-mail: burak-bilki@uiowa.edu
}

Forward calorimeters in CMS will need to be upgraded for the High Luminosity LHC (HL-LHC) operations, which is planned to be started in 2025. The major challenge is to preserve/improve the high performance of the current forward detectors while designing the detectors considerably radiation hard. This report will concentrate on the need for the upgrade, major challenges and various proposed R\&D concepts suitable for the Phase II upgrade framework. Various designs will be discussed with recent information about the beam tests and laboratory measurements.

Technology and Instrumentation in Particle Physics 2014,

2-6 June, 2014

Amsterdam, the Netherlands

\footnotetext{
* Speaker.

${ }^{\dagger}$ On behalf of the CMS Collaboration
} 


\section{Introduction}

The Compact Muon Solenoid (CMS) [1] is a general-purpose detector designed to run at the highest luminosity provided by the CERN Large Hadron Collider (LHC). The CMS detector calorimeter has been designed to detect the diverse signatures of new physics through the measurement of jets with moderate precision and by measuring missing transverse energy flow. The barrel and end-cap calorimeters are located inside the $4 \mathrm{~T}$ superconducting solenoidal magnet of length $13 \mathrm{~m}$ and inner diameter $5.9 \mathrm{~m}$.

The CMS electromagnetic calorimeter (ECAL) is a homogeneous crystal calorimeter and consists of a barrel (EB) and two endcaps (EE) made in total of $\sim 76 \mathrm{k} \mathrm{PbWO}_{4}$ crystals, with a 3 $\mathrm{X}_{0}\left(\mathrm{X}_{0}\right.$ : radiation lengths) lead - silicon strip preshower (ES) detector in the endcap section. The scintillation light is read-out by a pair of avalanche photodiodes (APDs) for each EB crystal and a vacuum phototriode for each EE crystal. The small Moliere radius $(\sim 2 \mathrm{~cm})$ in combination with the large number of crystals results in a fine granularity for the lateral shower shape. In the forward region, the granularity is further improved by the preshower detector (ES) $1.65<|\eta|<2.6$ ( $\eta$ : pseudorapidity) which consists of 2 orthogonal planes of silicon strips. Further information about the ECAL can be found in [2-6].

The hadron calorimeter (HCAL) is used to measure the hadronic showers. The cylindrically symmetric hadron barrel (HB) calorimeter consisting of alternating layers of brass and plastic scintillator plates surrounds the lead tungstate electromagnetic calorimeter (EB). The HB design maximizes the number of interaction lengths inside the coil, which requires minimizing the amount of space devoted to the active medium. The scintillator tiles are read out with embedded wavelength shifting (WLS) fibers. Brass was chosen as the absorber material because it is non-magnetic. This design made construction relatively simple, lending itself to projective tower geometry, and eliminated uninstrumented gaps. The CMS HCAL contains 9072 readout channels organized into four subsystems: barrel (HB, 2592 channels), endcap (HE, 2592 channels), outer (HO, 2160 channels) and forward (HF, 1728 channels). The performance of the HB, HE, HO, and HF were also extensively investigated and are reported in [2, 6-9].

Between 2010 and 2013, the LHC was operated with protons at beam energies of 3.5 and 4 $\mathrm{TeV}$. The proton beams consisted of single bunches and trains of $150 \mathrm{~ns}$ (2010), $75 \mathrm{~ns}$ (2011) and $50 \mathrm{~ns}$ (2011 and 2012) bunch spacing. Performances well beyond what had been expected initially have been achieved with 50 ns beams, culminating in the discovery of a $125 \mathrm{GeV} / \mathrm{c} 2$ Higgs boson by the ATLAS and CMS experiments [10,11]. The original design bunch spacing of $25 \mathrm{~ns}$ was only used for electron-cloud scrubbing runs at injection and for collision tests and future operation. The cycle structure evolved over the years, and the operational $\beta^{*}$ (betatron oscillation amplitude at the interaction point) for ATLAS and CMS was lowered in steps from $3.5 \mathrm{~m}$ (2010) to $0.6 \mathrm{~m}$ (2012). An overview of LHC operation between 2010 and 2013, so called Run 1, is provided in ref. [12].

Currently, the LHC is in the Long Shutdown 1 (LS1) period and the CMS along with the LHC and other experiments is going through various upgrade processes (see e.g. [13]). The start-up in 2015 , Run 2, is aimed at collision energy of $13 \mathrm{TeV}$ and an instantaneous luminosity at the nominal value i.e. $1 \times 10^{34} \mathrm{~cm}^{-2} \mathrm{~s}^{-1}$. The performance reach is specified in terms of levelled luminosity. This implies that the peak luminosity that is virtually required to reach such a leveled luminosity is much higher. More information about the levelling approaches can be found in [14]. 
At the end of Run 2, LHC is planned to reach the collision energy of $14 \mathrm{TeV}$ and instantaneous luminosity of $1.6 \times 10^{34} \mathrm{~cm}^{-2} \mathrm{~s}^{-1}$. Nominal bunch spacing of $25 \mathrm{~ns}$ will be used starting this run period. The expected average pileup will be around 40 . The target integrated luminosity before Long Shutdown 2 (LS2) is $\sim 200 \mathrm{fb}^{-1}$.

During Run 3, which will start after LS2 in 2020, LHC will reach 2x nominal luminosity, again in a levelled approach. The collision energy and the bunch spacing parameters will be at their nominal values, $14 \mathrm{TeV}$ and $25 \mathrm{~ns}$ respectively. The projected average pileup will be $\sim 50$. Run 3 is aimed at an integrated luminosity of $500 \mathrm{fb}^{-1}$.

Long Shutdown 3 (LS3) between 2023-2025 is the planned time interval for the significant detector upgrades necessary for the operations beyond 2025. Following LS3, in Run 4, the LHC will operate in the High-Luminosity LHC (HL-LHC) regime. This will be realized by instantaneous luminosities between 5 to 10 times the nominal luminosity at the nominal collision energy and bunch spacing. The target integrated luminosity in the HL-LHC era is $\sim 3000 \mathrm{fb}^{-1}$.

Here we report on the necessity of CMS forward calorimeter upgrades for the HL-LHC era and describe the possible upgrade options considered by CMS. The forward region is considered to be $1.4<|\eta|<5$. The upgrade detectors described are planned to be installed during LS3.

\section{Performance Projections of the CMS Forward Calorimeters}

The ECAL Endcap detectors, covering $1.4<|\eta|<3$, will experience progressive deterioration of energy resolution and trigger efficiency with strong dependence on the pseudorapidity. Figure 1 (left) shows the fraction of the ECAL response at various operating conditions to the nominal response of the ECAL as a function of pseudorapidity. The EE detectors should be replaced for optimal operation in the HL-LHC era.
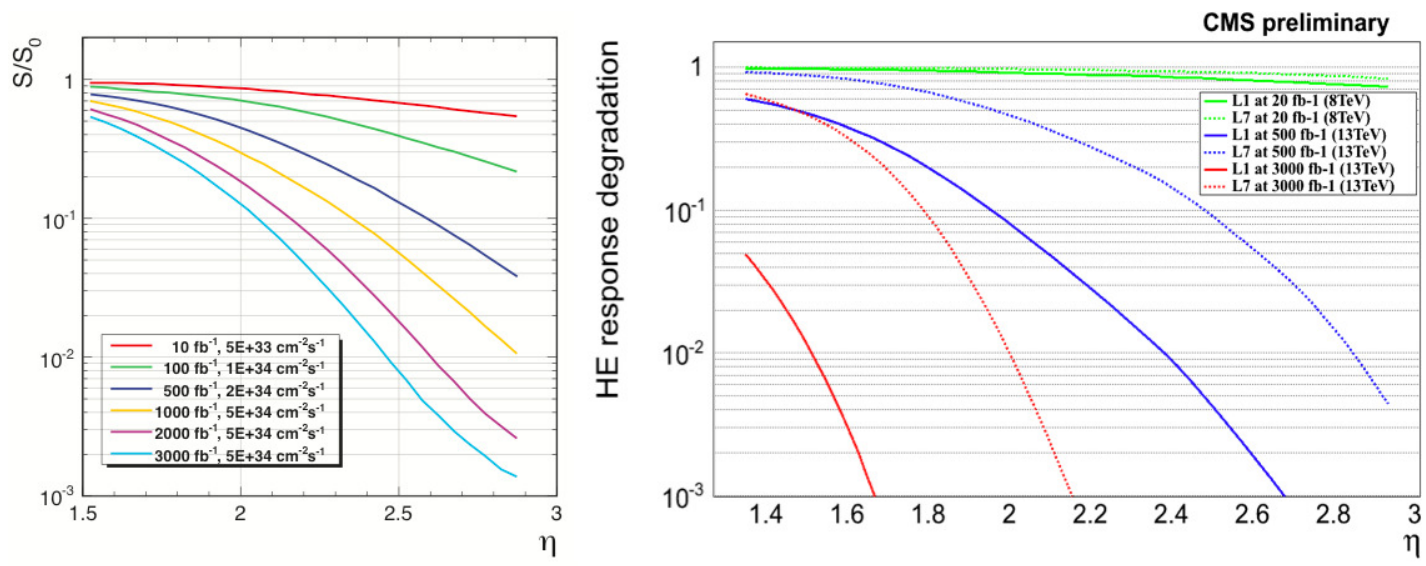

Figure 1: Simulated fraction of ECAL response to $50 \mathrm{GeV}$ electrons under different operating conditions as a function of pseudorapidity (left) and response degradation of the Hadron Endcap calorimeters at different operating points for two different longitudinal segmentations in the calorimeter and as a function of pseudorapidity (right).

Figure 1 (right) shows the response degradation of the Hadron Endcap calorimeters for different conditions and as a function of the pseudorapidity. HE covers $1.3<|\eta|<3$ and L1 and L7 
represent the longitudinal samplings at 1 and 7 layer depths. The green solid and dashed lines are based on the data acquired between 2010 and 2013, $500 \mathrm{fb}^{-1}$ and $3000 \mathrm{fb}^{-1}$ lines (blue and red, solid and dashed) are predictions based on the measurements. The performance degradation dictates that the HE detectors will need to be upgraded/replaced for the HL-LHC running conditions.

The coverage between $3<|\eta|<5$ is provided by the Hadron Forward (HF) calorimeters. The HF calorimeters will be sufficiently performant through HL-LHC and there is no upgrade planned by CMS.

\section{Concepts Considered for Phase II Upgrade}

There are two options considered by CMS for the Phase II Detector Upgrade: Shashlik electromagnetic calorimeter + HE rebuild; High Granularity Calorimeter.

\subsection{Shashlik EE + HE Rebuild}

This option is based on replacing the EE + ES with the shashlik design ECAL and replacing the active media of the HE and extending the longitudinal and transverse segmentation of the calorimeter.

The expected performance of various EE replacement detectors has been studied for long and of these, an architecture based upon W absorber layers and LYSO sampling layers read out with quartz capillaries with waveshifter cores and GaInP photosensors has been found to potentially provide the best electromagnetic energy resolution $\sim 10 \% / \sqrt{E}$ in the absence of radiation damage and pileup.

Figure 2 shows the design of the basic shashlik module. A single module consists of 282.5 $\mathrm{mm}$ thick W plates and $291.5 \mathrm{~mm}$ thick LYSO crystals both having $14 \mathrm{~mm}$ lateral sizes. The length of a single module is $114 \mathrm{~mm}$. The readout is provided by 4 wavelength shifting quartz capillaries, which are coupled to 1 or $2 \mathrm{GaInP}$ photodetectors. The module also consists of a calibration fiber running through the center. The shashlik modules have shorter length and smaller transverse size resulting in a larger number of modules. The modules also have smaller Moliere radius and radiation length. There are also substantial differences in terms of the light yield and temperature dependence. Table 1 shows the comparison of the parameters of shashlik EE and $\mathrm{PbWO}_{4} \mathrm{EE}$.

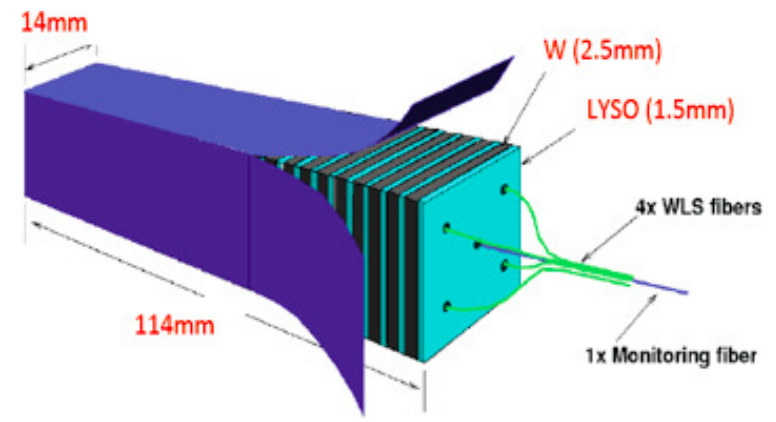

Figure 2: Design of the basic shashlik module. 
Table 1: Parametric comparison of the shashlik EE and $\mathrm{PbWO}_{4} \mathrm{EE}$.

\begin{tabular}{|l|c|c|}
\hline & W/LYSO(Ce) & PbWO $_{4}$ \\
\hline Length (mm) & 114 & 220 \\
\hline Transverse Size (mm) & 14 & 28.6 \\
\hline Detector Modules & 60,800 & 14,648 \\
\hline Moliere Radius (mm) & 13.7 & 21 \\
\hline Radiation Length - X \\
(mm) & 5.1 & 8.9 \\
\hline Light Yield (Relative to NaI) & 85 & 0.3 \\
\hline Emission Wavelength (nm) & 420 & 420 \\
\hline Decay Time (ns) & 40 & 25 \\
\hline Light Output (pe/MeV) & 6 & 4.5 \\
\hline Temperature Dependence (\%/C) & -0.2 & -2.2 \\
\hline
\end{tabular}

The major advantages of LYSO are brightness and density. Stability of the scintillation mechanism and material costs are the outstanding $\mathrm{R} \& \mathrm{D}$ issues. Other crystals, in particular $\mathrm{CeF}_{3}$, are being investigated. The merits of $\mathrm{CeF}_{3}$ include its lighter components that lead to less hadronic radiation damage at a given dose and recovery from hadronic radiation damage (unlike LYSO in which hadronic radiation damage is cumulative). In addition, the scintillation time constant is shorter (below $30 \mathrm{~ns}$ versus $40 \mathrm{~ns}$ for LYSO); the peak emission is in the ultraviolet (310-340 nm versus $430 \mathrm{~nm}$ for LYSO) and so will require a different wavelength shifter that emits in the 400 to $500 \mathrm{~nm}$ range. Finally, $\mathrm{CeF}_{3}$ is relatively more insensitive to temperature compared to LYSO.

The current options for wavelength shifting capillaries are thick quartz wall and quartz rod. The thick quartz wall capillary has an outer diameter of $1.2 \mathrm{~mm}$ and an inner diameter of $0.4 \mathrm{~mm}$. The core is a liquid wavelength shifter with the ends plugged. Photodetector is coupled to the quartz annulus and the far end is mirrored. The quartz rod has $1 \mathrm{~mm}$ diameter with powdered wavelength shifter on the outer surface as a thin layer. The wavelength shifter for both cases is envisaged as J2 (Y11) or DSB1 with other alternatives possible. The major advantage of the quartz capillaries is radiation hardness of both quartz and the wavelength shifter. The $R \& D$ requirements are the irradiation tests and stability studies of various options and test beam measurements. Ceriumdoped quartz fibers are also considered among alternatives.

It has been shown that photodetectors with similar performance to SiPMs with Ga compounds, specifically GaAs, can be fabricated. The development of photosensors from GaInP that have the potential of being 200,000 times as radiation hard as Si based photosensors is underway. The fabrication of SPADs (single photon avalanche devices) made from GaInP is completed and the SPADs achieved the expected low dark currents of $1 \mathrm{pA}$. It is worth pointing out that GaInP is not considered to be an "exotic" material in the semiconductor industry and indeed, is used along with GaAs in many applications. It now appears that this will be the leading contender for the shashlik EE.

The proposed readout scheme for EE is built upon existing strategies i.e. this can be made compatible with the readout scheme foreseen for the ECAL barrel at Phase II or with the QIE10 
electronics aimed for $\mathrm{HE}$ at Phase I.

The two potential roles of HE rebuild in the Phase II upgrade are the fully functioning hadron calorimeter behind shashlik EE and the backing hadron calorimeter of the HGC (high granularity calorimeter). In this context and in the view of the HL-LHC conditions, the proposed R\&D paths for the HE rebuild can be summarized as follows:

- Study of radiation damage to the CMS HE and of possible alternative solutions

- Radiation study of present system

- Development and radiation damage study of finger tiles, liquid scintillator tiles and green emitting scintillators

- Finger scintillator option for HE upgrade beyond Phase I

- Understanding of radiation damage of present tiles at $30 \mathrm{fb}^{-1}$

- Radiation damage of present tiles up to $7 \mathrm{Mrad}\left(700 \mathrm{fb}^{-1}\right)$

- Radiation damage of finger tiles up to $25 \operatorname{Mrad}\left(3000 \mathrm{fb}^{-1}\right)$

- Crystal fiber R\&D

- Layer of LuAG crystal fibers coupled to quartz capillaries.

- Development of radiation-hard WLS Films, Tiles, and Fibers

- Quartz plate R\&D

- Development of UV-absorbing WLS fibers

While following these $R \& D$ paths, the mechanical design will also be studied to enable larger longitudinal and lateral coverage.

\subsection{High Granularity Calorimeter (HGC)}

A high granularity calorimeter with a detailed sampling in both the hadronic and the electromagnetic sections with excellent pointing capability could have significant performance benefits. The approach that has been developed is a sampling calorimeter with layers of silicon detectors that feature very high longitudinal and lateral granularities in the electromagnetic and the front hadronic calorimeter sections, and a coarser segmentation backing hadronic calorimeter section which is under the development framework of the HE rebuild option.

The major components of the HGC are summarized below:

- Electromagnetic Calorimeter:

- 30 samplings of lead/copper total of $25 \mathrm{X}_{0}$

* 10 layers of $0.5 \mathrm{X}_{0} / 10$ layers of $0.8 \mathrm{X}_{0} / 10$ layers of $1.2 \mathrm{X}_{0}$

* Pad size $0.9 \mathrm{~cm}^{2}$ for first 20 layers, $1.8 \mathrm{~cm}^{2}$ for the last 10 layers

- $420 \mathrm{~m}^{2}$ of silicon pad detectors 
- 3.7M channels

- Front Hadronic Calorimeter:

- 4 interaction lengths

- 12 layers of brass/silicon each 0.33 interaction lengths

- Pad size is $1.8 \mathrm{~cm}^{2}$

- $1.4 \mathrm{M}$ channels

- Backing Calorimeter:

- Five interaction lengths (e.g. sampling of 0.5 interaction lengths)

- Since the radiation levels are lower, plastic scintillators can be used as active medium

The HGC is envisaged to have an unprecedented granularity resulting in 5.1M readout channels utilizing $670 \mathrm{~m}^{2}$ of Silicon as the active medium. Table 2 shows the parameters of the electromagnetic and front hadron calorimeter sections of the HGC.

Table 2: Parameters of the electromagnetic (EE) and front hadron (FH) calorimeter sections of HGC.

\begin{tabular}{|l|c|c|c|}
\hline & EE & FH & Total \\
\hline Area of Silicon $\left.\mathbf{( m}^{\mathbf{2}}\right)$ & 420 & 250 & 670 \\
\hline Channels & $3.7 \mathrm{M}$ & $1.4 \mathrm{M}$ & $5.1 \mathrm{M}$ \\
\hline Detector Modules & $19 \mathrm{~K}$ & $11 \mathrm{~K}$ & $30 \mathrm{~K}$ \\
\hline Weight One Endcap (tonnes) & 16 & 63 & 79 \\
\hline Number of Plates & 30 & 12 & 42 \\
\hline Front-end Power $(\mathbf{k W})$ & $70-80$ & $20-30$ & $90-110$ \\
\hline
\end{tabular}

All Silicon sensors will be made with the standard $320 \mu \mathrm{m}$ thick wafers. For regions of low radiation, $300 \mu \mathrm{m}$ depletion depth is foreseen. The medium and high radiation regions will be equipped with $200 \mu \mathrm{m}$ and $100 \mu \mathrm{m}$ depletion length sensors respectively.

The front-end electronics design for HGC utilizes an input capacitor of $50-100 \mathrm{pF}$, a time constant of the order of 15-20 ns and a single MIP response larger than 15k e- before damage. Onchip digitization for each channel will be at $40 \mathrm{MHz}$. The sum of 4 (2) adjacent pads will serve as the Level 1 Trigger primitives with 8-10 bit resolution. The target power consumption per channel is $15 \mathrm{~mW}$.

\section{Beam Tests}

The CMS Forward Calorimetry Upgrade groups have already started the test beam effort in December 2013 at Fermilab Test Beam Facility (FTBF) [15, 16]. The groups perform their tests under the collaboration of Fermilab T-1041 "CMS Forward Calorimetry R\&D" Experiment [17]. All groups are planning on future beam tests at Fermilab and CERN as well as irradiation tests at CERN and other non-HEP facilities. 


\section{Summary}

The CMS endcap electromagnetic and hadron calorimeters will experience significant performance degradation due to high radiation environment exposed by the High Luminosity LHC conditions and will need to be replaced/upgraded during Long Shutdown 3. In this context, CMS considers two upgrade scenarios:

- Shashlik EE + HE Rebuild: The endcap electromagnetic calorimeter as well as the endcap preshower detector will be replaced by the shashlik type electromagnetic calorimeter, and the hadron endcap calorimeter will be improved in terms of active media and lateral and longitudinal coverage.

- High Granularity Calorimeter: A high granularity calorimeter based on Silicon as the active medium will be constructed in two sections with varying segmentation - electromagnetic and front hadronic. The backing hadron calorimeter with a coarser segmentation will be built with the HE rebuild options.

Both scenarios have rigorous $R \& D$ plans on active media (e.g. scintillators, silicon sensors, crystals), readout components (e.g. fibers and photodetectors) and readout electronics (e.g. ondetector electronics).

\section{References}

[1] S. Chatrchyan et al. (CMS collabration), JINST 3 S08004, 2008.

[2] G. L. Bayatian et al. (CMS collabration),CERN/LHCC-2006-001, 2006.

[3] P. Adzic et al. (CMS ECAL Collaboration), Eur. Phys. J. C 44 1-10, 2006.

[4] P. Adzic et al. (CMS ECAL Collaboration), JINST 2 P04004, 2007.

[5] S. Chatrchyan et al. (CMS Collaboration), JINST 8 S09009, 2013.

[6] S. Abdullin et al. (CMS ECAL and HCAL Collaborations), Eur. Phys. J. C 60 359-373, 2009.

[7] S. Abdullin et al. (CMS HCAL Collaboration), Eur. Phys. J. C 55 159-171, 2008.

[8] S. Abdullin et al. (CMS HCAL Collaboration), Eur. Phys. J. C 53 139-166, 2008.

[9] G.L. Bayatian et al. (CMS Collaboration), CMS-NOTE-2008/010, 2008.

[10] G. Aad G et al. (ATLAS Collaboration), Phys. Lett. B 716 1-29, 2012.

[11] S. Chatrchyan et al. (CMS Collaboration), Phys. Lett. B 716 30-61, 2012.

[12] R. Alemany-Fernandez et al., CERN-ACC-NOTE-2013-0041, 2013.

[13] B. Bilki and Y. Onel, CMS Hadron Forward Calorimeter Phase I Upgrade Status, poster presented at this conference.

[14] M. Giovannozzi, AIP Conference Proceedings 1560 686, 2013.

[15] http://www-ppd.fnal.gov/FTBF/

[16] A. Soha, Improvements to the Fermilab Test Beam Facility, talk given at this conference.

[17] http://www-ppd.fnal.gov/FTBF/TSW/PDF/T1041_tsw_signed.pdf 